\title{
Assessment of Oropharyngeal Dysphagia in Patients With Parkinson Disease: Use of Ultrasonography
}

\author{
Eun Hyun Oh, MD, Jin Seok Seo, MD, Hyo Jung Kang, MD
}

Department of Physical Medicine and Rehabilitation, Veterans Health Service Medical Center, Seoul, Korea

\begin{abstract}
Objective To compare tongue thickness, the shortest hyoid-thyroid approximation (distance between the hyoid bone and thyroid cartilage), and the time interval between the initiation of tongue movement and the time of the shortest hyoid-thyroid approximation, by using ultrasonography in healthy controls and patients with Parkinson disease (PD).

Methods Healthy controls and PD patients with dysphagia were compared. Ultrasonography was performed 3 times for the evaluation of tongue thickness, the shortest hyoid-thyroid approximation, and the time between the initiation of tongue movement and the shortest hyoid-thyroid approximation.

Results A total of 24 healthy controls and 24 PD patients with dysphagia were enrolled. No significant differences were demonstrated between the two groups for the shortest hyoid-thyroid approximation (controls, $1.19 \pm 0.34$ $\mathrm{cm}$; PD patients, $1.37 \pm 0.5 \mathrm{~cm}$; $=0.15$ ) and tongue thickness (controls, $4.42 \pm 0.46 \mathrm{~cm}$; PD patients, $4.27 \pm 0.51 \mathrm{~cm}$; $\mathrm{p}=0.3$ ). In contrast, the time to the shortest hyoid-thyroid approximation was significantly different between the two groups (controls, $1.53 \pm 0.87 \mathrm{~ms}$; PD patients, $2.4 \pm 1.4 \mathrm{~ms}, \mathrm{p}=0.048$ ).

Conclusion Ultrasonography can be useful in evaluating dysphagia in patients with PD by direct visualization and measurement of the hyoid bone. Moreover, ultrasonography might contribute to a greater understanding of the pathophysiology of dysphagia in PD.
\end{abstract}

Keywords Parkinson disease, Dysphagia, Ultrasonography, Hyoid bone, Tongue

Received July 20, 2015; Accepted August 20, 2015

Corresponding author: Hyo Jung Kang

Department of Physical Medicine and Rehabilitation, Veterans Health

Service Medical Center, 53 Jinhwangdo-ro 61-gil, Gangdong-gu, Seoul 05368, Korea

Tel: +82-2-2225-1399, Fax: +82-2-2225-1579, E-mail: khojing@hanmail. net

(c) This is an open-access article distributed under the terms of the Creative Commons Attribution Non-Commercial License (http://creativecommons. org/licenses/by-nc/4.0) which permits unrestricted noncommercial use, distribution, and reproduction in any medium, provided the original work is properly cited.

Copyright $\odot 2016$ by Korean Academy of Rehabilitation Medicine

\section{INTRODUCTION}

Parkinson disease (PD) is a common neurodegenerative disorder resulting from the progressive loss of dopamine-generating cells in the ventrolateral portion of the pars compacta in the substantia nigra of the midbrain. A patient with PD may first experience movement-related symptoms, including tremor at rest, rigidity, akinesia (or bradykinesia), and postural instability [1], as well as bulbar dysfunction in the form of dysphagia, dysarthria, hypophonia, and sialorrhea. Non-motor symptoms, au- 
tonomic dysfunction, sleep disturbance and behavioral, cognitive and psychiatric problems may also arise as the disease progresses [1-3].

Dysphagia is a common feature of bulbar dysfunction in PD, with prevalence ranging from $16 \%$ to $55 \%$ [4], and is significantly associated with longer disease duration and dementia, as well as a greater occurrence in males [5]. In the majority of cases, the pathophysiology of dysphagia in PD is related to oral and pharyngeal phase dysfunction, characterized by abnormal bolus formation, multiple tongue elevation, delayed swallowing reflex, and decreased elevation and excursion of the hyolaryngeal complex [6,7]. Furthermore, a nutritional imbalance resulting from poor food intake and aspiration pneumonia can occur, and is the major cause of death in patients with PD [8]. Although there is a high risk of aspiration and pneumonia associated with dysphagia, the dysphagia is often overlooked until the patient experiences pneumonia. Therefore, it is important to evaluate swallowing function before problems arise. To evaluate dysphagia in a clinical setting, a videofluoroscopic swallowing study (VFSS) has been recommended. VFSS can be performed with a variety of foods and drinks containing barium, regardless of the patient's age and pathophysiology, including central nervous system or oropharyngeal pathophysiology [9].

Recent efforts have been made to evaluate dysphagia using ultrasonography (US). US is utilized in many fields of medicine, and has the benefits of low cost, absence of radiation exposure, and ease of accessibility. Moreover, US can directly and dynamically evaluate a structure with movement. Kuhl et al. [10] suggested that laryngeal elevation, the most important process in swallowing and a determinant factor for aspiration or penetration, could be measured easily by identification of the hyoid bone and the thyroid cartilage. Since then, a number of reports have used US to evaluate tongue thickness, tongue movement, and hyoid bone movement in healthy controls or stroke patients [11-14]. These reports have shown that hyoid bone movement, a major process of swallowing needed to prevent aspiration, can be measured by US as the distance between the thyroid cartilage and hyoid bone $[13,14]$. However, to our knowledge, although US has been used to evaluate stroke patients, there have been no reports of US being used to evaluate dysphagia in patients with PD [14,15].
The primary aim of this study was to use US in healthy controls and PD patients to compare the shortest hyoidthyroid distance, tongue thickness, and the time interval between the initiation of tongue movement and the shortest hyoid-thyroid approximation. The secondary aim was to demonstrate whether the above US parameters were correlated with PD severity and VFSS findings.

\section{MATERIALS AND METHODS}

\section{Participants}

The study was approved by the ethics committee of our hospital, and informed consent was obtained from each participant. From January 2014 to April 2015, healthy controls and a consecutive series of PD patients with dysphagia were enrolled in this study prospectively. Inclusion criteria for the PD group were as follows: patients 1) with a confirmed diagnosis of PD by a neurologist, 2) with subjective clinical complaints of dysphagia, including slow eating; food, liquid and tablet swallowing difficulties; food sticking in the throat; cough and wet voice after solid/liquid intake; nocturnal sialorrhea; or slow eating [16], 3) with stable disease, 'on state', with the same medications for more than 2 months, and 4) able to perform 3-step commands. Exclusion criteria for the PD group were as follows: participants 1) with severe cognitive impairment, 2) with other neurologic disorders that can induce dysphagia, such as stroke, traumatic brain injury, or motor neuron disease, 3) with a history of other conditions associated with oropharyngeal structures, such as thyroidectomy, vocal cord palsy, or cancer, and 4) with difficulty sustaining the evaluation process or with difficulty applying the US probe to the neck due to a Levin tube or tracheostomy. Medical records data, including age, gender, body mass index (BMI), MiniMental Status Examination (MMSE), and Modified Barthel Index (MBI) were recorded in both groups. Duration of PD and a modified Hoehn-Yahr (HY) scale score were also recorded [17].

\section{Ultrasonographic evaluation}

An ultrasound machine (Accuvix XQ; Medison, Seoul, Korea) was used with a 2-6 MHz curved-array transducer. The evaluations were performed by one investigator (EH Oh), and were repeated three times for all participants. Throughout the process, the least pressure 
with a large amount of coupling agent was applied on the probe to minimize the effect of the transducer on swallowing movements. The participants were examined in a comfortable upright sitting position with a neutral head position. The transducer was placed in the midsagittal plane on the submental area (Fig. 1A, 1B), which showed a longitudinal view from the tongue base to the thyroid cartilage on a monitor. The participants were asked not to move their head or neck and to maintain a relaxed state, and then the maximum tongue thickness was measured (Fig. 1C). Two anatomic references, the hyoid bone and thyroid cartilage, were used for measuring the approximate hyoid-thyroid distance [10]. This can be visualized as two calcifications, each with an acoustic shadow, indicating ossification of the hyoid bone and thyroid cartilage (Fig. 1C). The distance between the two calcifications is the hyoid-thyroid approximation. The investigator asked the participants to swallow $0.5 \mathrm{~mL}$ of water, and simultaneously began to record a video loop with a program for the US equipment. Three swallows were recorded for each participant with intervals of 1 minute. The shortest hyoid-thyroid approximation distance for each swallow was determined by tracing the recorded images and then measuring with an electronic caliper in the US equipment (Fig. 1D). In addition, the frame counts between the initiation of tongue movement and the shortest hyoidthyroid approximation were investigated and calculated in milliseconds, and this was considered the time to hyoid-thyroid approximation.

\section{Videofluoroscopic swallowing study}

Of 24 patients with PD, 15 were evaluated by VFSS in an upright seated position. Fluoroscopy (Sonialvision Versa; Shimadzu, Kyoto, Japan) was performed with a lateral view and recorded on VHS cassettes in real time. The VFSS protocol included the following: 1) water intake, 2) puree intake, and 3) semisolid intake (cookies with $5 \mathrm{~mL}$ of $20 \%$ semisolid or liquid containing barium material) [18].

For VFSS, the focus of our evaluation was on penetration and aspiration, which both occur during the pharyngeal phase. Penetration refers to when the intake bolus remains above the true vocal folds, inferior to the epiglottis and spilling into the laryngeal vestibule. Aspiration refers to materials that remain below the level of the vocal folds before the initiation of swallowing. If the food did not pass into the true vocal fold, it was considered to be penetration, and if the food passed into the true vocal fold, it was considered to be aspiration [18]. The PD patients were then divided into two subgroups based on the presence of penetration or aspiration during VFSS.

\section{Statistical analysis}

The intra-rater reliability of all US parameters, includ-
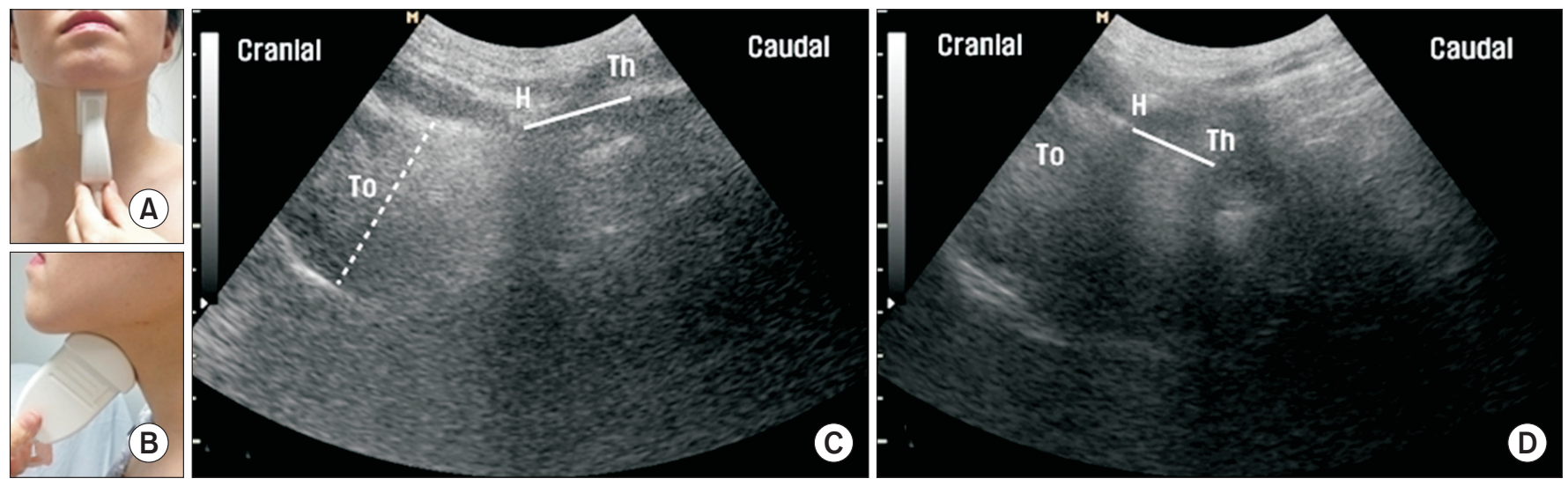

Fig. 1. Longitudinal ultrasonographic images of hyoid-thyroid approximation at rest and during the swallowing state. (A) Longitudinal approach of ultrasonographic probe, anterior view. (B) Longitudinal approach of ultrasonographic probe, lateral view. (C) At rest, the thickest area of the tongue ('To') is shown by the dotted line, and the hyoid-thyroid approximation, which is the distance between the hyoid bone (' $\left.\mathrm{H}^{\prime}\right)$ and the thyroid cartilage ('Th'), is shown by the solid line. (D) During swallowing, hyoid-thyroid approximation occurs with laryngeal elevation. The shortest distance of hyoid-thyroid approximation is shown by the solid line. 
ing tongue thickness, shortest hyoid-thyroid approximation, and time to hyoid-thyroid approximation, were analyzed by determining the intraclass correlation coefficient (ICC) of 3 trials for each parameter. An independent ttest was used to compare the baseline characteristics (age, gender, BMI, height, weight, MMSE score, and MBI score) of healthy controls and PD patients, and to compare the shortest hyoid-thyroid approximation distance and tongue thickness between the two groups. Time to hyoidthyroid approximation was analyzed with the MannWhitney U test. Spearman rank correlation analysis was performed in order to assess the correlation between US parameters and disease severity, as measured by PD duration, MBI scores, MMSE scores, and the HY scale scores; and also to assess the correlation between each of the US parameters. In addition, PD patients were divided into two subgroups: patients with or without penetration or aspiration during VFSS. US parameters in the two subgroups were compared with the Mann-Whitney U test. All statistical analyses were performed using SPSS ver. 17.0 for Windows (SPSS Inc., Chicago, IL, USA). The level of statistical significance was set at a p-value less than 0.05 .

\section{RESULTS}

A total of 48 participants (24 healthy controls and $24 \mathrm{PD}$ patients, all men) were enrolled in this study. The baseline characteristics of the study population are presented in Table 1. There was a significant difference in MBI scores (controls, 94.96 \pm 10.29 ; PD patients, $72.83 \pm 27.37$; $\mathrm{p}=0.001$ ) and MMSE scores (controls, 29.67 \pm 0.76 ; PD patients, 24.46 $\pm 7.48 ; \mathrm{p}=0.002$ ), which can be explained by the disease properties of PD. There were no significant differences between the groups in other characteristics. The mean HY scale score of PD patients was $2 \pm 1.54$ (10 patients were stage 1 ; one patient was stage 1.5 ; three patients were stage 2 ; two patients were stage 3 ; five patients were stage 4 ; and three patients were stage 5).

The intra-rater ICC of tongue thickness, the shortest hyoid-thyroid approximation, and time to hyoid-thyroid approximation were $0.777,0.896$, and 0.766 , respectively (mean=0.813), indicating good reproducibility.

No significant differences were demonstrated between healthy controls and PD patients for the shortest hyoidthyroid approximation (controls, $1.19 \pm 0.34 \mathrm{~cm}$; PD patients, $1.37 \pm 0.5 \mathrm{~cm}$; $\mathrm{p}=0.149$, independent $\mathrm{t}$-test) and tongue thickness (controls, $4.42 \pm 0.46 \mathrm{~cm}$; PD patients, $4.27 \pm 0.51 \mathrm{~cm} ; \mathrm{p}=0.292$, independent t-test). However, the time to the shortest hyoid-thyroid approximation was significantly different between the two groups (controls, $1.53 \pm 0.87 \mathrm{~ms}$; PD patients, $2.4 \pm 1.4 \mathrm{~ms} ; \mathrm{p}=0.048$, MannWhitney U test) (Table 2).

According to Spearman rank correlation analysis, the time to hyoid-thyroid approximation, which was significantly different between the two groups, was positively

Table 1. Demographic characteristics of the subjects

\begin{tabular}{lccl}
\hline \multicolumn{1}{c}{ Variable } & $\begin{array}{c}\text { Control } \\
(\mathbf{n = 2 4})\end{array}$ & $\begin{array}{c}\text { PD } \\
(\mathbf{n = 2 4})\end{array}$ & p-value \\
\hline Gender (all men) & 24 & 24 & 1.00 \\
Age (yr) & $66.71 \pm 12.97$ & $71.67 \pm 5.10$ & 0.088 \\
MMSE & $29.67 \pm 0.76$ & $24.46 \pm 7.48$ & $0.002^{*}$ \\
MBI & $94.96 \pm 10.29$ & $72.83 \pm 27.37$ & $0.001^{*}$ \\
BMI & $25.02 \pm 2.95$ & $23.65 \pm 4.00$ & 0.182 \\
Height (cm) & $166.79 \pm 6.22$ & $165.75 \pm 6.96$ & 0.587 \\
Weight (kg) & $69.96 \pm 11.42$ & $65.19 \pm 12.47$ & 0.174 \\
Onset of PD (yr) & - & $7.21 \pm 5.96$ & \\
HY scale & - & $2 \pm 1.54$ & \\
$\quad$ Stage $1 / 1.5 / 2 /$ & & $10 / 1 / 3 / 2 / 5 / 3$ & \\
3/4/5 & & & \\
\hline
\end{tabular}

Values are presented as mean \pm standard deviation or number.

BMI, body mass index; HY scale, modified Hoehn and Yahr scale; MBI, Modified Barthel Index; PD, Parkinson disease; MMSE, Mini-Mental State Examination.

${ }^{*} \mathrm{p}<0.05$.

Table 2. Comparison of ultrasonographic parameters in healthy controls and PD patients

\begin{tabular}{lccc}
\hline \multicolumn{1}{c}{ Variable } & $\begin{array}{c}\text { Control } \\
(\mathbf{n = 2 4})\end{array}$ & $\begin{array}{c}\text { PD } \\
(\mathbf{n = 2 4})\end{array}$ & p-value \\
\hline Tongue thickness (cm) & $4.42 \pm 0.46$ & $4.27 \pm 0.51$ & 0.292 \\
HT approximation (cm) & $1.19 \pm 0.34$ & $1.37 \pm 0.5$ & 0.149 \\
$\begin{array}{c}\text { Time to HT } \\
\text { approximation (ms) }\end{array}$ & $1.53 \pm 0.87$ & $2.4 \pm 1.4$ & $0.048^{\mathrm{a})}$ \\
\hline
\end{tabular}

Values are presented as mean \pm standard deviation.

HT approximation, the shortest distance of hyoid-thyroid approximation; PD, Parkinson disease; Time to HL approximation, time interval between the initiation of tongue movement and the shortest distance of hyoidthyroid approximation.

${ }^{a)} \mathrm{p}<0.05$ by Mann-Whitney $U$ test. 
correlated with PD duration ( $\mathrm{r}=0.467, \mathrm{p}=0.033)$ and negatively correlated with MBI $(\mathrm{r}=-0.437, \mathrm{p}=0.003)$ and MMSE $(\mathrm{r}=-0.308, \mathrm{p}=0.047)$. However, there was no correlation between time to hyoid-thyroid approximation and $\mathrm{HY}$ scale score $(\mathrm{r}=0.341, \mathrm{p}=0.142)$. There were no significant correlations among the three US parameters in this study.

Finally, PD patients were divided into two subgroups based on the presence of penetration or aspiration during VFSS $(n=6)$ or not $(n=9)$, and their US parameters were analyzed with the Mann-Whitney U test. However, no significant differences were found between the two subgroups.

\section{DISCUSSION}

Dysphagia is an important problem in PD because it can cause aspiration pneumonia and thereby increase the mortality rate [3]. There have been many attempts to evaluate dysphagia in PD using VFSS, and they have revealed impairment in the oral and pharyngeal phases. Such impairment includes inappropriate tongue movement, piecemeal deglutition, a delayed swallowing reflex and oropharyngeal transit time, residues in the vallecular space and pyriform fossa, and penetration or aspiration $[3,6,7,19]$. Dysphagia assessment using US is a new technique that has been proposed to have many advantages, including lack of radiation, noninvasiveness, and the possibility for use with bedridden patients. In addition, it allows for visualization of the anatomic structure of the tongue and dynamic movement of the hyoid bone. Furthermore, the reliability of US for analyzing hyoid bone displacement was proved with a good single-measure ICC by 3 raters: an inter-rater agreement of 0.86 for rest measurements (prior to swallowing) and 0.86 for maximal hyoid displacement measurements [11]. Intra-rater reliability was also excellent, at 0.95 for rest measurements, and 0.98 for maximal displacement measurements [11].

Early studies evaluating dysphagia using US focused mainly on tongue movement [20]. Later, hyoid bone displacement, the most important process of swallowing, was evaluated quantitatively and dynamically [13-15,21]. Kuhl et al. [10] first suggested that the laryngeal elevation following the hyoid bone movement could be identified by US as the approximation of two structures, the hyoid bone and the thyroid cartilage. In addition, there have been several research studies on hyoid-thyroid approximation in stroke patients, and these reports revealed that the hyoid-larynx approximation was lower in stroke patients with dysphagia than in those without dysphagia. It was explained that stroke in corticobulbar fibers, the medullary solitary tract nucleus and nucleus ambiguus structures, induced the weakness of pharyngeal and laryngeal muscles, which resulted in a decrease in the hyoid-larynx approximation $[14,15]$. However, there has been no attempt to evaluate dysphagia using US in PD. Therefore, this study focused on dysphagia evaluation through the use of US in patients with PD.

In contrast to the above results from studies with stroke patients, the present study demonstrated no significant differences of hyoid-thyroid approximation and tongue thickness, but instead, a significant difference in the time to hyoid-thyroid approximation in PD patients as compared with healthy controls (Table 2). Most dysphagia in patients with PD is related to oropharyngeal swallowing dysfunction, specifically lingual bradykinesia, movement hesitation, and tremor in the tongue [8]. In PD patients, there is a significantly delayed interval between the initiation of tongue movement and the shortest distance of hyoid-thyroid approximation, supporting the premise that dysphagia in PD is mainly a result of lingual dyskinesia in the oropharyngeal phase. Moreover, ultrasonographic examination contributed to these movements of the tongue and laryngopharynx complex.

Second, there was no significant correlation between the severity of PD, as assessed by HY grade, and US parameters, while the time to hyoid-thyroid approximation was positively correlated with duration in $\mathrm{PD}$, and negatively with MBI and MMSE. On the basis that HY scoring mainly reflects dysfunction in limbs, these results suggest that dysphagia in PD is closely related with disease progression, but not correlated with limb dysfunction. Many studies using VFSS found no correlation between the severity of PD and dysphagia [7,22,23]. The lack of correlation between limb muscle dysfunction and dysphagia in PD has been explained by variable submental muscle pathology, which have been demonstrated to have a higher percentage of atrophic myofibers and fast to slow fiber type transition, as well as decreased pharyngeal sensation specifically $[22,24]$. Although this study was limited by the small number of participants in each HY grade, these results also suggest that the pathophysiology 
of dysphagia may differ from that of limb muscles in PD.

Third, there was no influence of aspiration or penetration as assessed by VFSS on the US parameters. The small sample sizes for each subgroup (penetration or aspiration subgroup, $\mathrm{n}=6$; no penetration or aspiration subgroup, $\mathrm{n}=9$ ) are thought to be insufficient for statistical analysis. Although there was no significant difference between the two subgroups of PD patients according to VFSS findings, correlations between VFSS and US findings in PD patients should be evaluated in future studies to determine the diagnostic ability of US.

This study has some limitations. First, a small number of patients were enrolled, especially those who underwent VFSS. Second, most of the PD patients enrolled in this study had non-severe PD. Indeed, ultrasonographic examinations require participant's cooperation to follow 3-step commands and sustain a sitting position. Despite the fact that most of the enrolled patients had a relatively good functional reservoir, the results of this study showed a significant difference between PD patients and controls, which could support the reliability of the results.

In conclusion, by direct visualization and evaluation of the hyoid bone, US provides a useful way in which to evaluate dysphagia in patients with PD. Moreover, it may contribute to a greater understanding of the pathophysiology of dysphagia in PD.

\section{CONFLICT OF INTEREST}

No potential conflict of interest relevant to this article was reported.

\section{REFERENCES}

1. Jankovic J. Parkinson's disease: clinical features and diagnosis. J Neurol Neurosurg Psychiatry 2008;79:36876.

2. Storch A, Schneider CB, Wolz M, Sturwald Y, Nebe A, Odin $\mathrm{P}$, et al. Nonmotor fluctuations in Parkinson disease: severity and correlation with motor complications. Neurology 2013;80:800-9.

3. Nicaretta DH, Rosso AL, Mattos JP, Maliska C, Costa MM. Dysphagia and sialorrhea: the relationship to Parkinson's disease. Arq Gastroenterol 2013;50:42-9.

4. Kalf JG, de Swart BJ, Bloem BR, Munneke M. Prevalence of oropharyngeal dysphagia in Parkinson's disease: a meta-analysis. Parkinsonism Relat Disord 2012;18:311-5.

5. Cereda E, Cilia R, Klersy C, Canesi M, Zecchinelli AL, Mariani CB, et al. Swallowing disturbances in Parkinson's disease: a multivariate analysis of contributing factors. Parkinsonism Relat Disord 2014;20:1382-7.

6. Baijens LW, Speyer R, Passos VL, Pilz W, Roodenburg N, Clave P. Swallowing in Parkinson patients versus healthy controls: reliability of measurements in videofluoroscopy. Gastroenterol Res Pract 2011;2011:380682.

7. Monte FS, da Silva-Junior FP, Braga-Neto P, Nobre e Souza MA, de Bruin VM. Swallowing abnormalities and dyskinesia in Parkinson's disease. Mov Disord 2005;20:457-62.

8. Troche MS, Okun MS, Rosenbek JC, Musson N, Fernandez $\mathrm{HH}$, Rodriguez R, et al. Aspiration and swallowing in Parkinson disease and rehabilitation with EMST: a randomized trial. Neurology 2010;75:1912-9.

9. Logemann JA. Role of the modified barium swallow in management of patients with dysphagia. Otolaryngol Head Neck Surg 1997;116:335-8.

10. Kuhl V, Eicke BM, Dieterich M, Urban PP. Sonographic analysis of laryngeal elevation during swallowing. J Neurol 2003;250:333-7.

11. Macrae PR, Doeltgen SH, Jones RD, Huckabee ML. Intra- and inter-rater reliability for analysis of hyoid displacement measured with sonography. J Clin Ultrasound 2012;40:74-8.

12. Kim JH, Kim MS. Lateral pharyngeal wall motion analysis using ultrasonography in stroke patients with dysphagia. Ultrasound Med Biol 2012;38:2058-64.

13. Yabunaka K, Sanada H, Sanada S, Konishi H, Hashimoto T, Yatake H, et al. Sonographic assessment of hyoid bone movement during swallowing: a study of normal adults with advancing age. Radiol Phys Technol 2011;4:73-7.

14. Huang YL, Hsieh SF, Chang YC, Chen HC, Wang TG. Ultrasonographic evaluation of hyoid-larynx approximation in dysphagic stroke patients. Ultrasound Med Biol 2009;35:1103-8.

15. Hsiao MY, Chang YC, Chen WS, Chang HY, Wang TG. Application of ultrasonography in assessing oropharyngeal dysphagia in stroke patients. Ultrasound Med Biol 2012;38:1522-8.

16. Volonte MA, Porta M, Comi G. Clinical assessment 
of dysphagia in early phases of Parkinson's disease. Neurol Sci 2002;23 Suppl 2:S121-2.

17. Goetz CG, Poewe W, Rascol O, Sampaio C, Stebbins GT, Counsell C, et al. Movement Disorder Society Task Force report on the Hoehn and Yahr staging scale: status and recommendations. Mov Disord 2004;19:10208.

18. Morgan A, Ward E, Murdoch B, Bilbie K. Acute characteristics of pediatric dysphagia subsequent to traumatic brain injury: videofluoroscopic assessment. J Head Trauma Rehabil 2002;17:220-41.

19. Nagaya M, Kachi T, Yamada T, Igata A. Videofluorographic study of swallowing in Parkinson's disease. Dysphagia 1998;13:95-100.

20. Shawker TH, Sonies B, Hall TE, Baum BF. Ultrasound analysis of tongue, hyoid, and larynx activity during swallowing. Invest Radiol 1984;19:82-6.

21. Fanucci A, Cerro P, Ietto F, Brancaleone C, Berardi F. Physiology of oral swallowing studied by ultrasonography. Dentomaxillofac Radiol 1994;23:221-5.

22. Mu L, Sobotka S, Chen J, Su H, Sanders I, Adler CH, et al. Altered pharyngeal muscles in Parkinson disease. J Neuropathol Exp Neurol 2012;71:520-30.

23. Umemoto G, Tsuboi Y, Kitashima A, Furuya H, Kikuta T. Impaired food transportation in Parkinson's disease related to lingual bradykinesia. Dysphagia 2011;26:250-5.

24. Mu L, Sobotka S, Chen J, Su H, Sanders I, Nyirenda $\mathrm{T}$, et al. Parkinson disease affects peripheral sensory nerves in the pharynx. J Neuropathol Exp Neurol 2013;72:614-23. 